\section{Electrochemistry of Porous Materials}

by Antonio Doménech-Carbó, CRC Press, Boca Raton, FL, USA, 2010, pp xxxviii + 312, ISBN 978-1-4398-0633-3. Price: GBP 82.00, USD 129.95

Porous materials are of interest for a range of applications: as supports for catalysts, energy conversion and storage and chromatography are amongst the most topical areas of interest currently. This book focuses on electrochemical aspects of porous materials. The book is well laid out, with 12 chapters written in a concise and focussed manner. Chapter 1 provides an introduction to electrochemical techniques, with sufficient information for researchers who are not familiar with electrochemistry while not delving into too much detail. Researchers interested in such detail can refer to the list of references provided. One minor editorial comment, capacity should be replaced by capacitance (p. 16) and nonelectron conducting materials by insulators (p. 27). Chapter 2 consists of a theoretical description of the electrochemical processes of porous materials. Chapter 3 describes the modelling of mechanisms of electrocatalysis.

The next chapters describe a range of materials used to prepare porous electrodes. Chapter 4 describes zeolites and aluminosilicates. The author uses Maya Blue as an interesting example of how electrochemical methods can be utilised to characterise and provide detailed information on microporous materials. Chapter 5 focuses on metal-organic frameworks and Chapter 6 on the electrochemical properties of porous oxides and layered hydrogels, including electrocatalytic studies. This is followed by a description of porous carbon and carbon nanotubes which are of interest for use as materials for hydrogen storage and in lithium batteries (Chapter 7). The electrochemistry of porous polymers and hybrid materials, including composite materials is described in Chapter 8 .

Subsequent chapters focus on applications, electrochemical sensing (Chapter 9), supercapacitors, batteries and fuel cells (Chapter 10), magnetoelectrochemical and photoelectrochemical applications (Chapter (11), culminating in a final brief chapter on electrosynthesis and environmental remediation.

The book, as expected from its title, focuses on electrochemical studies of porous materials; it does not describe any chromatographic applications which may be of interest to readers of Chromatographia (this is not a criticism, this reviewer is not aware of any such applications). A description of possible uses of porous materials in terms of commercial applications (or the potential for such applications) is not included in the book and would have been a useful addition.

Overall the book is a good reference volume for any researcher interested in the electrochemistry of porous materials; it provides a survey of virtually all of the current areas of interest in this field. The reference list is extensive and up to date and is of interest to anybody interested in obtaining detailed information on a particular topic. It would make a good textbook for a specialist graduate level course.

Edmond Magner 